\title{
Low Energy Asymptotics of the Spectral Shift Function for Pauli Operators with Nonconstant Magnetic Fields
}

\author{
by
}

Georgi D. RAIKov

\begin{abstract}
We consider the 3D Pauli operator with nonconstant magnetic field $\mathbf{B}$ of constant direction, perturbed by a symmetric matrix-valued electric potential $V$ whose coefficients decay fast enough at infinity. We investigate the low-energy asymptotics of the corresponding spectral shift function. As a corollary, for generic negative $V$, we obtain a generalized Levinson formula, relating the low-energy asymptotics of the eigenvalue counting function and of the scattering phase of the perturbed operator.
\end{abstract}

2010 Mathematics Subject Classification: 35J10, 81Q10, 35P20, 35P25, 47F05.

Keywords: Pauli operators, spectral shift function, low-energy asymptotics, Levinson formula.

\section{$\S 1$. Introduction}

Suppose that the magnetic field $\mathbf{B}: \mathbb{R}^{3} \rightarrow \mathbb{R}^{3}$ has a constant direction, say,

$$
\mathbf{B}=(0,0, b) \text {. }
$$

By the Maxwell equation, $\operatorname{div} \mathbf{B}=0$, we should then have $\partial b / \partial x_{3}=0$. Assume that the function $b: \mathbb{R}^{2} \rightarrow \mathbb{R}$ is continuous and bounded. In Subsection 2.1 we describe in more detail the class of admissible functions $b$. Let $\mathbf{A} \in C^{1}\left(\mathbb{R}^{3} ; \mathbb{R}^{3}\right)$ be a magnetic potential generating the magnetic field $\mathbf{B}$, i.e. $\operatorname{curl} \mathbf{A}=\mathbf{B}$. Introduce the Pauli matrices

$$
\hat{\sigma}_{1}=\left(\begin{array}{ll}
0 & 1 \\
1 & 0
\end{array}\right), \quad \hat{\sigma}_{2}=\left(\begin{array}{cc}
0 & -i \\
i & 0
\end{array}\right), \quad \hat{\sigma}_{3}=\left(\begin{array}{cc}
1 & 0 \\
0 & -1
\end{array}\right) .
$$

Communicated by H. Okamoto. Received August 21, 2009.

G. D. Raikov: Facultad de Matemáticas, Pontificia Universidad Católica de Chile, Vicuña Mackenna 4860, Macul, Santiago de Chile, Chile;

e-mail: graikov@mat.puc.cl

(C) 2010 Research Institute for Mathematical Sciences, Kyoto University. All rights reserved. 
Set $\hat{\sigma}:=\left(\hat{\sigma}_{1}, \hat{\sigma}_{2}, \hat{\sigma}_{3}\right)$. Let

$$
H_{0}:=(\hat{\sigma} \cdot(-i \nabla-\mathbf{A}))^{2}
$$

be the unperturbed self-adjoint Pauli operator defined originally on $C_{0}^{\infty}\left(\mathbb{R}^{3} ; \mathbb{C}^{2}\right)$, and then closed in $L^{2}\left(\mathbb{R}^{3} ; \mathbb{C}^{2}\right)$. We have

$$
H_{0}:=\left(\begin{array}{cc}
(-i \nabla-\mathbf{A})^{2}-b & 0 \\
0 & (-i \nabla-\mathbf{A})^{2}+b
\end{array}\right)=:\left(\begin{array}{cc}
H_{0}^{-} & 0 \\
0 & H_{0}^{+}
\end{array}\right)=H_{0}^{-} \oplus H_{0}^{+} .
$$

Further, let $v_{j k} \in L^{\infty}\left(\mathbb{R}^{3}\right), j, k=1,2$. Assume that $v_{11}$ and $v_{22}$ are real-valued, and $v_{12}=\overline{v_{21}}$. Introduce the symmetric matrix

$$
V(\mathbf{x}):=\left(\begin{array}{ll}
v_{11}(\mathbf{x}) & v_{12}(\mathbf{x}) \\
v_{21}(\mathbf{x}) & v_{22}(\mathbf{x})
\end{array}\right), \quad \mathbf{x} \in \mathbb{R}^{3} .
$$

On the domain of $H_{0}$ define the operator

$$
H:=H_{0}+V \text {. }
$$

Assume that

$$
(H-i)^{-1}-\left(H_{0}-i\right)^{-1} \in S_{1}\left(L^{2}\left(\mathbb{R}^{3} ; \mathbb{C}^{2}\right)\right)
$$

where $S_{1}(X)$ denotes the trace class of linear operators acting in the Hilbert space $X$. By the diamagnetic inequality and the boundedness of $b$, we find that (1.3) holds true if

$$
\left|v_{j k}\right|^{1 / 2}(-\Delta+1)^{-1} \in S_{2}\left(L^{2}\left(\mathbb{R}^{3}\right)\right), \quad j, k=1,2,
$$

where $S_{2}(X)$ denotes the Hilbert-Schmidt class of linear operators acting in $X$. In its turn, 1.4 holds true if and only if $v_{j k} \in L^{1}\left(\mathbb{R}^{3}\right)$.

By (1.3), there exists a unique $\xi=\xi\left(\cdot ; H, H_{0}\right) \in L^{1}\left(\mathbb{R} ;\left(1+E^{2}\right)^{-1} d E\right)$ which vanishes identically on $(-\infty, \inf \sigma(H))$, such that the Lifshits-Krein trace formula

$$
\operatorname{Tr}\left(f(H)-f\left(H_{0}\right)\right)=\int_{\mathbb{R}} \xi\left(E ; H, H_{0}\right) f^{\prime}(E) d E
$$

holds for each $f \in C_{0}^{\infty}(\mathbb{R})$ (see the original works [25], [22], or [33, Chapter 8]). The function $\xi\left(\cdot ; H, H_{0}\right)$ is called the spectral shift function (SSF) for the operator pair $\left(H, H_{0}\right)$. If $E<0=\inf \sigma\left(H_{0}\right)$, then the spectrum of $H$ below $E$ could be at most discrete, and for almost every $E<0$ we have

$$
\xi\left(E ; H, H_{0}\right)=-N(E ; H)
$$

where $N(E ; H)$ denotes the number of eigenvalues of $H$ lying in the interval $(-\infty, E)$, and counted with their multiplicities. On the other hand, for almost 
every $E \in \sigma_{\mathrm{ac}}\left(H_{0}\right)=[0, \infty)$ (see Corollary 2.2), the $\operatorname{SSF} \xi\left(E ; H, H_{0}\right)$ is related to the scattering determinant $\operatorname{det} S\left(E ; H, H_{0}\right)$ for the pair $\left(H, H_{0}\right)$ by the BirmanKrein formula

$$
\operatorname{det} S\left(E ; H, H_{0}\right)=e^{-2 \pi i \xi\left(E ; H, H_{0}\right)}
$$

(see the original work [8] or [33, Section 8.4]).

A priori, the $\operatorname{SSF} \xi\left(E ; H, H_{0}\right)$ is defined for almost every $E \in \mathbb{R}$. In this article, if $E \in(-\infty, \mathcal{C}) \backslash\{0\}$ where $\mathcal{C}>0$ is a constant defined in 2.13), we will identify $\xi\left(E ; H, H_{0}\right)$ with a representative of its equivalence class, described explicitly in Subsection 4.1 under the assumption that the matrix $V(\mathbf{x}), \mathbf{x} \in \mathbb{R}^{3}$, is positive- or negative-semidefinite. Under our generic assumptions on $V$, we check that the SSF $\xi\left(\cdot ; H, H_{0}\right)$ is bounded on every compact subset of $(-\infty, \mathcal{C}) \backslash\{0\}$, and continuous on $(-\infty, \mathcal{C}) \backslash\left(\{0\} \cup \sigma_{\mathrm{pp}}(H)\right)$ where $\sigma_{\mathrm{pp}}(H)$ denotes the set of eigenvalues of $H$ (see Proposition 4.1.

The main results of the article concern the asymptotic behavior of the SSF $\xi\left(E ; H, H_{0}\right)$ as $E \rightarrow 0$ for perturbations $V$ of definite sign. We show that even for certain $V$ of compact support, the $\operatorname{SSF} \xi\left(\cdot ; H, H_{0}\right)$ has a singularity at the origin (see Theorems 3.1 and 3.2. More precisely, we show that $\xi\left(E ; H, H_{0}\right) \rightarrow \infty$ as $E \downarrow 0$ if the perturbation is positive, and $\xi\left(E ; H, H_{0}\right) \rightarrow-\infty$ as $E \uparrow 0$ and $E \downarrow 0$ if the perturbation is negative. The singularities of the SSF at the origin are described in terms of effective Hamiltonians of Berezin-Toeplitz type; their spectral properties have been studied, for instance, in [29], [31], and [30]. Assuming that the perturbation admits a power-like or exponential decay at infinity, or that it has a compact support, we obtain the first asymptotic term of $\xi\left(E ; H, H_{0}\right)$ as $E \uparrow 0$ and $E \downarrow 0$ (see Corollaries 3.6 and 3.7). In particular, if the perturbation is negative, we show that the limit

$$
\lim _{E \downarrow 0} \frac{\xi\left(E ; H, H_{0}\right)}{\xi\left(-E ; H, H_{0}\right)}
$$

exists, is finite and positive; it depends only on the decay rate of $V$ at infinity (see Corollary 3.8.

Similar results concerning the singularities at the Landau levels of the SSF in the case where the unperturbed operator is the 3D Schrödinger operator with constant magnetic field, and the perturbation is a scalar potential of constant sign which decays fast enough at infinity, were obtained in [15]. The relation between these singularities and the possible accumulation of resonances at the Landau levels was considered in 10 .

The paper is organized as follows. In Section 2 we discuss the class of admissible magnetic fields, describe the basic spectral properties of the operator $H_{0}$, 
and introduce the Berezin-Toeplitz operators we need. In Section 3 we formulate our main results as well as some corollaries. Section 4 is devoted to auxiliary material such as the representation of the SSF due to A. Pushnitski, and estimates of appropriate sandwiched resolvents. Finally, Section 5 contains the proofs of Theorems 3.1 and 3.2

\section{§2. Admissible magnetic fields and effective Hamiltonians}

\section{$\S 2.1$. Admissible magnetic fields}

Let $\mathbf{B}$ have the form (1.1). Assume that $b=b_{0}+\tilde{b}$ where $b_{0}>0$ is a constant, while the function $\tilde{b}: \mathbb{R}^{2} \rightarrow \mathbb{R}$ is such that the Poisson equation

$$
\Delta \tilde{\varphi}=\tilde{b}
$$

admits a solution $\tilde{\varphi}: \mathbb{R}^{2} \rightarrow \mathbb{R}$, continuous and bounded together with its derivatives up to the second order. Slightly abusing the terminology, we will say that $b$ is an admissible magnetic field. Also, we will call the constant $b_{0}$ the mean value of $b$, and $\tilde{b}$ the background of $b$. In our leading example, the admissible background $\tilde{b}$ has the form

$$
\tilde{b}(x)=\int_{\mathbb{R}^{2}} e^{i \lambda \cdot x} d \nu(\lambda), \quad x \in \mathbb{R}^{2}
$$

where $\nu$ is a Borel charge (i.e. a complex-valued measure) defined on $\mathbb{R}^{2}$ which satisfies

$$
\begin{aligned}
& |\nu|\left(\mathbb{R}^{2}\right)<\infty, \\
& \nu(\delta)=\overline{\nu(-\delta)}
\end{aligned}
$$

for each Borel set $\delta \subset \mathbb{R}^{2}$,

$$
\nu(\{0\})=0
$$

and

$$
\int_{\mathbb{R}^{2}}|\lambda|^{-2} d|\nu|(\lambda)<\infty
$$

If $\tilde{b}$ satisfies 2.2, then the Poisson equation 2.1 admits a solution

$$
\tilde{\varphi}(x):=-\int_{\mathbb{R}^{2}}|\lambda|^{-2} e^{i \lambda \cdot x} d \nu(\lambda), \quad x \in \mathbb{R}^{2},
$$

which possesses all the prescribed properties.

Let us give two further examples of admissible backgrounds $\tilde{b}$ of the form 2.2 . 
(i) Let $\lambda_{n} \in \mathbb{R}^{2} \backslash\{0\}, b_{n} \in \mathbb{C}, n \in \mathbb{N}$. Assume that $\sum_{n \in \mathbb{N}}\left|b_{n}\right|\left(1+\left|\lambda_{n}\right|^{-2}\right)<\infty$. Then the almost periodic function $\tilde{b}(x):=\sum_{n \in \mathbb{N}} b_{n} e^{i \lambda_{n} \cdot x}, x \in \mathbb{R}^{2}$, is an admissible background, provided that it is real-valued. In this case the charge $\nu$ in 2.2 is singular with respect to the Lebesgue measure in $\mathbb{R}^{2}$. Evidently, the real-valued periodic functions with zero mean value and absolutely convergent series of Fourier coefficients belong to the above defined class of admissible backgrounds.

(ii) Let $f: \mathbb{R}^{2} \rightarrow \mathbb{C}$ be a Lebesgue measurable function which satisfies $f(\lambda)=$ $\overline{f(-\lambda)}, \lambda \in \mathbb{R}^{2}$, and $\int_{\mathbb{R}^{2}}\left(1+|\lambda|^{-2}\right)|f(\lambda)| d \lambda<\infty$. Then $\tilde{b}(x):=\int_{\mathbb{R}^{2}} e^{i \lambda \cdot x} f(\lambda) d \lambda$ is again an admissible background. In this case the charge $\nu$ in 2.2 is absolutely continuous with respect to the Lebesgue measure in $\mathbb{R}^{2}$.

For $\left(x_{1}, x_{2}\right) \in \mathbb{R}^{2}$ set $\varphi_{0}:=b_{0}\left(x_{1}^{2}+x_{2}^{2}\right) / 4$ and

$$
\varphi:=\varphi_{0}+\tilde{\varphi},
$$

$\tilde{\varphi}$ being introduced in 2.1). Then $\Delta \varphi_{0}=b_{0}$ and $\Delta \varphi=b$. Put $\mathbf{A}:=\left(A_{1}, A_{2}, A_{3}\right)$ with

$$
A_{1}:=-\frac{\partial \varphi}{\partial x_{2}}, \quad A_{2}:=\frac{\partial \varphi}{\partial x_{1}}, \quad A_{3}=0 .
$$

The magnetic potential $\mathbf{A}:=\left(A_{1}, A_{2}, A_{3}\right) \in C^{1}\left(\mathbb{R}^{3}, \mathbb{R}^{3}\right)$ generates the magnetic field $\mathbf{B}=$ curl $\mathbf{A}=(0,0, b)$. Changing the gauge if necessary, we will assume that the magnetic potential $\mathbf{A}$ in $(1.2)$ is given by 2.9 .

\section{$\S 2.2$. Spectral properties of the operator $H_{0}$}

Introduce the the annihilation and the creation operators

$$
a=a(b):=-2 i e^{-\varphi} \frac{\partial}{\partial \bar{z}} e^{\varphi}, \quad a^{*}=a(b)^{*}:=-2 i e^{\varphi} \frac{\partial}{\partial z} e^{-\varphi},
$$

the function $\varphi$ being defined in (2.8), and $z:=x_{1}+i x_{2}, \bar{z}:=x_{1}-i x_{2}$. The operators $a$ and $a^{*}$ defined initially on $C_{0}^{\infty}\left(\mathbb{R}^{2}\right)$, and then closed in $L^{2}\left(\mathbb{R}^{2}\right)$, are mutually adjoint. Set

$$
\begin{gathered}
H_{\perp}^{-}=H_{\perp}^{-}(b):=a^{*} a, \quad H_{\perp}^{+}=H_{\perp}^{+}(b):=a a^{*}, \\
H_{\perp}=H_{\perp}(b):=\left(\begin{array}{cc}
H_{\perp}^{-} & 0 \\
0 & H_{\perp}^{+}
\end{array}\right)=H_{\perp}^{-} \oplus H_{\perp}^{+} .
\end{gathered}
$$

Then we have

$$
\begin{aligned}
& \operatorname{Ker} H_{\perp}^{-}=\operatorname{Ker} a=\left\{u \in L^{2}\left(\mathbb{R}^{2}\right) \mid u=g e^{-\varphi}, \frac{\partial g}{\partial \bar{z}}=0\right\}, \\
& \operatorname{Ker} H_{\perp}^{+}=\operatorname{Ker} a^{*}=\left\{u \in L^{2}\left(\mathbb{R}^{2}\right) \mid u=g e^{\varphi}, \frac{\partial g}{\partial z}=0\right\}, \\
& \operatorname{Ker} H_{\perp}=\left\{\mathbf{u}=\left(u_{1}, u_{2}\right) \mid u_{1} \in \operatorname{Ker} H_{\perp}^{-}, u_{2} \in \operatorname{Ker} H_{\perp}^{+}\right\} .
\end{aligned}
$$


Note that Ker $H_{\perp}^{-}$(respectively, Ker $H_{\perp}^{+}$) is a weighted holomorphic (respectively, antiholomorphic) space of Fock-Segal-Bargmann type (see e.g. 18, Section 2 and Subsection 3.2]). Since we have chosen $b_{0}>0$, and $\tilde{\varphi}$ is supposed to be bounded, we find that $\operatorname{dim} \operatorname{Ker} H_{\perp}^{-}=\infty$ while $\operatorname{dim} \operatorname{Ker} H_{\perp}^{+}=0$.

Proposition 2.1 ([30, Proposition 1.2]). Let $b$ be an admissible magnetic field with $b_{0}>0$. Then $0=\inf \sigma\left(H_{\perp}\right)$ is an isolated eigenvalue of infinite multiplicity. More precisely,

$$
\operatorname{dim} \operatorname{Ker} H_{\perp}=\infty,
$$

and

$$
(0, \mathcal{C}) \subset \mathbb{R} \backslash \sigma\left(H_{\perp}\right)
$$

with

$$
\mathcal{C}:=2 b_{0} \exp (-2 \operatorname{osc} \tilde{\varphi}),
$$

where osc $\tilde{\varphi}:=\sup _{x \in \mathbb{R}^{2}} \tilde{\varphi}(x)-\inf _{x \in \mathbb{R}^{2}} \tilde{\varphi}(x)$.

Remarks. (i) Relation 2.12 holds true also for more general backgrounds $\tilde{b}$. For example, it is sufficient that $\tilde{b}$ is bounded, and the solution $\tilde{\varphi} \in C^{2}\left(\mathbb{R}^{2}\right)$ of the Poisson equation 2.1 satisfies only

$$
\tilde{\varphi}(x)=o\left(|x|^{2}\right), \quad|x| \rightarrow \infty .
$$

If $\tilde{b}$ is of the form (2.2), and relations $(2.3)-2.5)$ (but not necessarily (2.6) hold, then

$$
\tilde{\varphi}(x):=\int_{\mathbb{R}^{2}} \frac{(\lambda \cdot x)^{2}}{|\lambda|^{2}} \int_{0}^{1}(1-s) e^{i s \lambda \cdot x} d s d \nu(\lambda), \quad x \in \mathbb{R}^{2},
$$

is in $C^{2}\left(\mathbb{R}^{2}\right)$, and satisfies 2.1 and (2.14). However, some of our further results, in particular, Lemma 2.3 below, may not be true for such more general magnetic fields.

(ii) If $b$ is a periodic magnetic field, the fact that the origin is an isolated eigenvalue of $H_{\perp}$ was already mentioned in [14, and was proved in [6]. A farreaching extension of the results of [14, concerning the existence of a strictly positive isolated eigenvalue of $H_{\perp}$ of infinite multiplicity, can be found in [26].

Now note that

$$
H_{0}^{ \pm}=H_{\perp}^{ \pm} \otimes I_{\|}+I_{\perp} \otimes H_{\|}
$$

where $I_{\|}$and $I_{\perp}$ are the identity operators in $L^{2}(\mathbb{R})$ and $L^{2}\left(\mathbb{R}^{2}\right)$ respectively, and

$$
H_{\|}:=-\frac{d^{2}}{d x_{3}^{2}}
$$


is the self-adjoint operator, originally defined on $C_{0}^{\infty}(\mathbb{R})$, and then closed in $L^{2}(\mathbb{R})$. Since $\sigma\left(H_{\|}\right)$coincides with $[0, \infty)$, and is purely absolutely continuous, while $\inf \sigma\left(H_{\perp}^{-}\right)=0$, we find that 2.15 combined with, say, the arguments of 2, Subsection 8.2.3] implies

Corollary 2.2. Assume that $b$ is an admissible magnetic field. Then the spectrum $\sigma\left(H_{0}\right)$ coincides with $[0, \infty)$, and is purely absolutely continuous.

\section{$\S 2.3$. Berezin-Toeplitz operators}

Denote by $p=p(b)$ the orthogonal projection onto $\operatorname{Ker} H_{\perp}^{-}(b)$ (see (2.10p). It is well known that $p$ admits a continuous integral kernel $\mathcal{P}_{b}(x, y), x, y \in \mathbb{R}^{2}$ (see e.g. [18, Theorem 2.3]).

Lemma 2.3. Assume that the magnetic field $b$ is admissible. Then

$$
\frac{b_{0}}{2 \pi} e^{-2 \operatorname{osc} \tilde{\varphi}} \leq \mathcal{P}_{b}(x, x) \leq \frac{b_{0}}{2 \pi} e^{2 \operatorname{osc} \tilde{\varphi}}, \quad x \in \mathbb{R}^{2} .
$$

Proof. Introduce the functions

$$
\phi_{k}(x):=\sqrt{\frac{b_{0}}{2 \pi k !}}\left(\frac{b_{0}}{2}\right)^{k / 2}\left(x_{1}+i x_{2}\right)^{k} e^{-\varphi_{0}(x)}, \quad k \in \mathbb{Z}_{+}, x \in \mathbb{R}^{2},
$$

which constitute a basis of $\operatorname{Ker} H_{\perp}^{-}\left(b_{0}\right)=\operatorname{Ker} a\left(b_{0}\right)$, orthonormal in $L^{2}\left(\mathbb{R}^{2}\right)$ (see e.g. 31]). Let $\gamma: l^{2}\left(\mathbb{Z}_{+}\right) \rightarrow l^{2}\left(\mathbb{Z}_{+}\right)$be the operator given in the canonical basis by the matrix $\left\{g_{j k}\right\}_{j, k=0}^{\infty}$ with $g_{j k}:=\int_{\mathbb{R}^{2}} e^{-2 \tilde{\varphi}} \phi_{j} \overline{\phi_{k}} d x, j, k \in \mathbb{Z}_{+}$. It is easy to see that $\gamma$ is self-adjoint, bounded, and

$$
\inf _{y \in \mathbb{R}^{2}} e^{-2 \tilde{\varphi}(y)} \leq \inf \sigma(\gamma) \leq \sup \sigma(\gamma) \leq \sup _{y \in \mathbb{R}^{2}} e^{-2 \tilde{\varphi}(y)} .
$$

Set $\rho:=\gamma^{-1 / 2}$. Let $\left\{r_{j k}\right\}_{j, k=0}^{\infty}$ be the matrix of $\rho$ in the canonical basis of $l^{2}\left(\mathbb{Z}_{+}\right)$. Put

$$
\psi_{j}(x):=e^{-\tilde{\varphi}(x)} \sum_{k=0}^{\infty} r_{j k} \phi_{k}(x), \quad x \in \mathbb{R}^{2}, j \in \mathbb{Z}_{+} .
$$

Then $\left\{\psi_{j}\right\}_{j=0}^{\infty}$ is a basis of Ker $a(b)$, orthonormal in $L^{2}\left(\mathbb{R}^{2}\right)$, and

$$
\mathcal{P}_{b}(x, x)=\sum_{j=0}^{\infty}\left|\psi_{j}(x)\right|^{2}=e^{-2 \tilde{\varphi}(x)}\|\rho \phi(x)\|_{l^{2}\left(\mathbb{Z}_{+}\right)}^{2}
$$

where $\phi(x):=\left\{\phi_{k}(x)\right\}_{k=0}^{\infty} \in l^{2}\left(\mathbb{Z}_{+}\right), x \in \mathbb{R}^{2}$ being fixed (see [18, Theorem 2.4]). Making use of 2.18) and the spectral theorem, we find that (2.19) and the obvious equality $\sum_{k=0}^{\infty}\left|\phi_{k}(x)\right|^{2}=\frac{b_{0}}{2 \pi}$, valid for each $x \in \mathbb{R}^{2}$, imply 2.16$)$. 
The Berezin-Toeplitz operators, necessary for the formulation of our main results, have the form $p(b) U p(b)$ where $U: \mathbb{R}^{2} \rightarrow \mathbb{R}$. In Lemma 2.4 below we describe a class of compact operators of this type (admitting also complex-valued $U$ ).

Let $X$ be a separable Hilbert space. In coherence with our previous notations $S_{1}(X)$ and $S_{2}(X)$, we denote by $S_{q}(X), q \in[1, \infty)$, the Schatten-von Neumann class of compact linear operators $T$ for which the norm $\|T\|_{q}:=\left(\operatorname{Tr}|T|^{q}\right)^{1 / q}$ is finite.

Lemma 2.4. Let $U \in L^{q}\left(\mathbb{R}^{2}\right), q \in[1, \infty)$. Assume that $b$ is an admissible magnetic field. Then $p(b) U p(b) \in S_{q}\left(L^{2}\left(\mathbb{R}^{2}\right)\right)$, and

$$
\|p(b) U p(b)\|_{q}^{q} \leq \frac{b_{0}}{2 \pi} e^{2 \operatorname{osc} \tilde{\varphi}}\|U\|_{L^{q}}^{q} .
$$

Proof. If $U \in L^{\infty}\left(\mathbb{R}^{2}\right)$, then

$$
\|p(b) U p(b)\| \leq\|U\|_{L^{\infty}}
$$

If $U \in L^{1}\left(\mathbb{R}^{2}\right)$, then by $p(b) U p(b)=p(b)|U|^{1 / 2} e^{i \arg U}|U|^{1 / 2} p(b)$ and $(2.16)$, we have

$$
\left\|e^{i \arg U}|U|^{1 / 2} p(b)\right\|_{2}^{2}=\left\|p(b)|U|^{1 / 2}\right\|_{2}^{2}=\int_{\mathbb{R}^{2}} \mathcal{P}_{b}(x, x)|U(x)| d x \leq \frac{b_{0}}{2 \pi} e^{2 \operatorname{osc} \tilde{\varphi}}\|U\|_{L^{1}}
$$

Therefore,

$$
\|p(b) U p(b)\|_{1} \leq \frac{b_{0}}{2 \pi} e^{2 \operatorname{osc} \tilde{\varphi}}\|U\|_{L^{1}} .
$$

Interpolating between 2.21 and 2.22 , we get 2.20 .

For further references, introduce the orthogonal projections

$$
P=P(b):=p \otimes I_{\|}, \quad Q=Q(b):=I-P,
$$

acting in $L^{2}\left(\mathbb{R}^{3}\right)$, and the orthogonal projections

$$
\mathbf{P}=\mathbf{P}(b):=\left(\begin{array}{cc}
P & 0 \\
0 & 0
\end{array}\right), \quad \mathbf{Q}=\mathbf{Q}(b):=\mathbf{I}-\mathbf{P}=\left(\begin{array}{cc}
Q & 0 \\
0 & I
\end{array}\right)
$$

acting in $L^{2}\left(\mathbb{R}^{3} ; \mathbb{C}^{2}\right)$. Here $I$ and $\mathbf{I}$ are the identity operators in $L^{2}\left(\mathbb{R}^{3}\right)$ and $L^{2}\left(\mathbb{R}^{3} ; \mathbb{C}^{2}\right)$ respectively. 


\section{§3. Main results}

\section{\$3.1. Statement of the main results}

For $\mathbf{x}=\left(x_{1}, x_{2}, x_{3}\right) \in \mathbb{R}^{3}$ we denote by $x=\left(x_{1}, x_{2}\right)$ the variables on the plane perpendicular to the magnetic field. Suppose that the matrix $V$ satisfies

$$
\begin{aligned}
v_{j k} \in C\left(\mathbb{R}^{3}\right), \quad\left|v_{j k}(\mathbf{x})\right| \leq C_{0}\langle x\rangle^{-m_{\perp}}\left\langle x_{3}\right\rangle^{-m_{3}}, \\
\mathbf{x}=\left(x, x_{3}\right) \in \mathbb{R}^{3}, j, k=1,2,
\end{aligned}
$$

with $C_{0}>0, m_{\perp}>2, m_{3}>1$, and $\langle y\rangle:=\left(1+|y|^{2}\right)^{1 / 2}, y \in \mathbb{R}^{d}, d \geq 1$. Our main results will be formulated under a more restrictive assumption than (3.1), namely

$$
v_{j k} \in C\left(\mathbb{R}^{3}\right), \quad\left|v_{j k}(\mathbf{x})\right| \leq C_{0}\langle\mathbf{x}\rangle^{-m}, \quad \mathbf{x} \in \mathbb{R}^{3}, j, k=1,2,
$$

with $m>3$. Note that $(3.2)$ implies $(3.1)$ with any $m_{3} \in(0, m)$ and $m_{\perp}=m-m_{3}$.

In what follows we will assume that the perturbation of the operator $H_{0}$ is of definite sign. For notational convenience, we will suppose that

$$
V(\mathbf{x}) \geq 0, \quad \mathbf{x} \in \mathbb{R}^{3},
$$

and will consider the operators $H_{0}+V$ or $H_{0}-V$.

Assume that (3.1) with $m_{\perp}>2, m_{3}>1$ and (3.3) hold true. Set

$$
W(x):=\int_{\mathbb{R}} v_{11}\left(x, x_{3}\right) d x_{3}, \quad x \in \mathbb{R}^{2} .
$$

If, moreover, $V$ satisfies 3.2 , then

$$
0 \leq W(x) \leq C_{0}^{\prime}\langle x\rangle^{-m+1}, \quad x \in \mathbb{R}^{2},
$$

where $C_{0}^{\prime}=C_{0} \int_{\mathbb{R}}\langle x\rangle^{-m} d x$. For $E>0$ introduce the operator

$$
\omega(E):=\frac{1}{2 \sqrt{E}} p(b) W p(b) .
$$

Evidently, $\omega(E)$ is self-adjoint and nonnegative in $L^{2}\left(\mathbb{R}^{2}\right)$. If $b$ is an admissible magnetic field, $E>0$, and $V$ satisfies 3.1 with $m_{\perp}>2$ and $m_{3}>1$, then Lemma 2.4 with $U=W$ implies $\omega(E) \in S_{1}$.

Let $T=T^{*}$. Denote by $\mathbb{P}_{\delta}(T)$ the spectral projection of $T$ associated with the Borel set $\delta \subset \mathbb{R}$. Suppose that $T$ is compact and put

$$
n_{ \pm}(s ; T):=\operatorname{rank} \mathbb{P}_{(s, \infty)}( \pm T), \quad s>0 .
$$

Our first theorem concerns the asymptotic behavior of the $\operatorname{SSF} \xi\left(E ; H, H_{0}\right)$ as the energy approaches the origin from below. 
Theorem 3.1. Let 3.2 with $m>3$ and 3.3 hold true. Assume that $b$ is an admissible magnetic field. Then for each $\varepsilon \in(0,1)$ we have

$$
\begin{aligned}
-n_{+}((1-\varepsilon) ; \omega(E))+O(1) & \leq \xi\left(-E ; H_{0}-V, H_{0}\right) \\
& \leq-n_{+}((1+\varepsilon) ; \omega(E))+O(1), \quad E \downarrow 0 .
\end{aligned}
$$

Remark. By (1.6), if (3.3) holds true, then $\xi\left(-E ; H_{0}+V, H_{0}\right)=0$ for each $E>0$.

Suppose again that the potential $V$ satisfies (3.1) with $m_{\perp}>2, m_{3}>1$, and (3.3. For $E>0$ define the matrix-valued function

$$
\mathcal{W}_{E}(x):=\left(\begin{array}{ll}
w_{11}(x) & w_{12}(x) \\
w_{21}(x) & w_{22}(x)
\end{array}\right), \quad x \in \mathbb{R}^{2},
$$

where

$$
\begin{aligned}
w_{11}(x) & :=\int_{\mathbb{R}} v_{11}\left(x, x_{3}\right) \cos ^{2}\left(\sqrt{E} x_{3}\right) d x_{3}, \\
w_{22}(x) & :=\int_{\mathbb{R}} v_{11}\left(x, x_{3}\right) \sin ^{2}\left(\sqrt{E} x_{3}\right) d x_{3}, \\
w_{12}(x)=w_{21}(x) & :=\int_{\mathbb{R}} v_{11}\left(x, x_{3}\right) \cos \left(\sqrt{E} x_{3}\right) \sin \left(\sqrt{E} x_{3}\right) d x_{3} .
\end{aligned}
$$

Set

$$
\Omega(E):=\frac{1}{2 \sqrt{E}} p(b) \mathcal{W}_{E} p(b)
$$

Evidently, $\Omega(E)$ is self-adjoint in $L^{2}\left(\mathbb{R}^{2} ; \mathbb{C}^{2}\right)$, and $\Omega(E) \geq 0$. Since $\omega(E) \in S_{1}$, it is easy to check that $\Omega(E) \in S_{1}$ as well.

Our second theorem concerns the asymptotic behavior of the $\operatorname{SSF} \xi\left(E ; H, H_{0}\right)$ as the energy approaches zero from above.

Theorem 3.2. Let 3.2 with $m>3$ and 3.3 hold true. Assume that $b$ is an admissible magnetic field. Then for each $\varepsilon \in(0,1)$ we have

$$
\begin{aligned}
& \pm \frac{1}{\pi} \operatorname{Tr} \arctan \left((1 \pm \varepsilon)^{-1} \Omega(E)\right)+O(1) \\
& \quad \leq \xi\left(E ; H_{0} \pm V, H_{0}\right) \leq \pm \frac{1}{\pi} \operatorname{Tr} \arctan \left((1 \mp \varepsilon)^{-1} \Omega(E)\right)+O(1), \quad E \downarrow 0 .
\end{aligned}
$$

Remark. The privileged role of the entry $v_{11}$ of the matrix $V$ which occurs in the operators $\omega(E)$ and $\Omega(E)$, is determined by our assumption that $b_{0}>0$, and hence, the kernel of $H_{\perp}$ consists of elements with spin-up polarization (see (2.11). In particular, we have

$$
\mathbf{P}(b) V \mathbf{P}(b)=\left(\begin{array}{cc}
P(b) v_{11} P(b) & 0 \\
0 & 0
\end{array}\right)
$$


The proofs of Theorems 3.1 and 3.2 can be found in Section 4. In the following subsection we will describe explicitly the asymptotics of $\xi\left(-E ; H_{0}-V, H_{0}\right)$ and $\xi\left(E ; H_{0} \pm V H_{0}\right)$ as $E \downarrow 0$, under generic assumptions about the behavior of $W(x)$ as $|x| \rightarrow \infty$.

\section{$\S 3.2$. Corollaries}

By (3.7) and (3.10), we can reduce the analysis of the behavior as $E \rightarrow 0$ of $\xi\left(E ; H_{0} \pm V, H_{0}\right)$ to the investigation of the eigenvalue asymptotics of compact Berezin-Toeplitz operators $p(b) U p(b)$, discussed in the following three lemmas.

The first one treats the case where the decay of $U$ at infinity is power-like. It involves the concept of an integrated density of states (IDS) for the operator $H_{\perp}^{-}(b)$. Let $\chi_{Q}$ be the characteristic function of the square $Q \subset \mathbb{R}^{2}$, and let $|Q|$ denote its area. We recall that the nonincreasing function $\varrho_{b}: \mathbb{R} \rightarrow[0, \infty)$ is called an IDS for the operator $H_{\perp}^{-}(b)$ if it satisfies

$$
\varrho_{b}(E)=\lim _{|Q| \rightarrow \infty}|Q|^{-1} \operatorname{Tr}\left(\chi_{Q} \mathbb{P}_{(-\infty, E)}\left(H_{\perp}^{-}(b)\right) \chi_{Q}\right)
$$

at its continuity points $E \in \mathbb{R}$ (see e.g. [20, 13]). If $b=b_{0}$, i.e. if $\tilde{b}=0$, we have

$$
\varrho_{b_{0}}(E)=\frac{b_{0}}{2 \pi} \sum_{q=0}^{\infty} \Theta\left(E-2 b_{0} q\right), \quad E \in \mathbb{R},
$$

where

$$
\Theta(t)= \begin{cases}0 & \text { if } t<0 \\ 1 & \text { if } t>0\end{cases}
$$

is the Heaviside function.

Lemma 3.3 ([30, Proposition 3.5]). Let $U \in C^{1}\left(\mathbb{R}^{2}\right)$ satisfy

$$
0 \leq U(x) \leq C_{1}\langle x\rangle^{-\alpha}, \quad|\nabla U(x)| \leq C_{1}\langle x\rangle^{-\alpha-1}, \quad x \in \mathbb{R}^{2},
$$

for $\alpha>0$ and $C_{1}>0$. Assume, moreover, that:

- $U(x)=u_{0}(x /|x|)|x|^{-\alpha}(1+o(1))$ as $|x| \rightarrow \infty$, where $u_{0}$ is a continuous function on $\mathbb{S}^{1}$ which does not vanish identically;

- $b$ is an admissible magnetic field;

- there exists an IDS $\varrho_{b}$ for the operator $H_{\perp}^{-}(b)$.

Then

$$
\begin{aligned}
n_{+}(s ; p(b) U p(b)) & =\frac{b_{0}}{2 \pi}\left|\left\{x \in \mathbb{R}^{2} \mid U(x)>s\right\}\right|(1+o(1)) \\
& =\Psi_{\alpha}\left(s ; u_{0}, b_{0}\right)(1+o(1)), \quad s \downarrow 0,
\end{aligned}
$$


where, as above, $|\cdot|$ denotes the Lebesgue measure, and

$$
\Psi_{\alpha}(s)=\Psi_{\alpha}\left(s ; u_{0}, b_{0}\right):=s^{-2 / \alpha} \frac{b_{0}}{4 \pi} \int_{\mathbb{S}^{1}} u_{0}(\theta)^{2 / \alpha} d \theta, \quad s>0 .
$$

Remarks. (i) In [30, Proposition 3.5] we considered only the example of almost periodic admissible magnetic fields, and proved explicitly the existence of the IDS for the operator $H_{\perp}^{-}(b)$. In Lemma 3.3 above the existence of the IDS is just a hypothesis. For this reason we summarize here the main ingredients of the proof of [30, Proposition 3.5] which do not concern the existence of the IDS:

- Applying variational and commutator techniques developed, in particular, in [12, 21], we show that for each $E \in(0, \mathcal{C})$,

$$
n_{+}(s ; p(b) U p(b))=n_{-}\left(s / E ; U^{1 / 2}\left(H_{\perp}^{-}-E\right)^{-1} U^{1 / 2}\right)(1+o(1)), \quad s \downarrow 0 .
$$

- Using the Birman-Schwinger principle, as well as the methods of [1, 23, 19] concerning the strong-electric-field asymptotics of the discrete spectrum of the operator $H_{\perp}^{-}(b)+g U$ lying in the gap $(0, \mathcal{C})$ of $\sigma\left(H_{\perp}^{-}(b)\right)$, we obtain

$$
\begin{aligned}
\lim _{g \rightarrow \infty} g^{-2 / \alpha} n_{-}\left(g^{-1}\right. & \left.; U^{1 / 2}\left(H_{\perp}^{-}-E\right)^{-1} U^{1 / 2}\right) \\
& =\int_{-\infty}^{E}\left|\left\{\left.x \in \mathbb{R}^{2}\left|u_{0}(x /|x|)\right| x\right|^{-\alpha}>E-t\right\}\right| d \varrho_{b}(t) \\
& =E^{-2 / \alpha} \frac{\mathcal{J}(b)}{2} \int_{\mathbb{S}^{1}} u_{0}(\theta)^{2 / \alpha} d \theta, \quad E \in(0, \mathcal{C}),
\end{aligned}
$$

where $\mathcal{J}(b)$ is the jump of the IDS $\varrho_{b}$ at the origin.

- We check that the family $H_{\perp}^{-}\left(b_{0}+s \tilde{b}\right), s \in[0,1]$, is continuous in the norm resolvent sense, and, utilizing a gap-labelling theorem due to J. Bellissard [5, Proposition 4.2.5], we find that the jump $J\left(b_{0}+s \tilde{b}\right)$ is independent of $s \in[0,1]$. In particular, 3.12 implies

$$
\mathcal{J}(b)=\mathcal{J}\left(b_{0}\right)=\frac{b_{0}}{2 \pi} .
$$

Putting together 3.15-(3.17), we obtain (3.13). As a by-product of (3.11) with any $E \in(0, \mathcal{C})$, and $(3.17)$, we obtain the formula

$$
\lim _{|Q| \rightarrow \infty}|Q|^{-1} \int_{Q} \mathcal{P}_{b}(x, x) d x=\frac{b_{0}}{2 \pi},
$$

valid if $b$ is an admissible magnetic field, and there exists an IDS $\varrho_{b}$ for the operator $H_{\perp}^{-}(b)$.

(ii) In the case $b=b_{0}$ (i.e. $\tilde{b}=0$ ) a variant of Lemma 3.3 was proved in 29. with the help of pseudo-differential techniques. In the case of general admissible 
backgrounds $\tilde{b}$, the methods of 29] are not directly applicable: due to the factor $\exp (-\tilde{\varphi})$ whose derivatives generically do not decay at infinity, we do not obtain suitable symbols of pseudo-differential operators.

The following two lemmas concern respectively the cases where $U$ decays exponentially at infinity, or has a compact support. First note that, by [30, Proposition 3.2], we have

$$
\begin{aligned}
n_{+}\left(\exp (2 \operatorname{osc} \tilde{\varphi}) s ; p\left(b_{0}\right) U p\left(b_{0}\right)\right) & \leq n_{+}(s ; p(b) U p(b)) \\
& \leq n_{+}\left(\exp (-2 \operatorname{osc} \tilde{\varphi}) s ; p\left(b_{0}\right) U p\left(b_{0}\right)\right),
\end{aligned}
$$

provided that $s>0, U: \mathbb{R}^{2} \rightarrow[0, \infty)$, and the operator $U(-\Delta+1)^{-1}$ is compact in $L^{2}\left(\mathbb{R}^{2}\right)$.

Combining (3.18) with the results of [31, Proposition 3.1 with $q=0$ ] and of [31, Proposition 3.2], we obtain

Lemma 3.4. Let $0 \leq U \in L^{\infty}\left(\mathbb{R}^{2}\right)$. Assume that

$$
\ln U(x)=-\eta|x|^{2 \beta}(1+o(1)), \quad|x| \rightarrow \infty,
$$

for some $\beta, \eta \in(0, \infty)$. Let $b$ be an admissible magnetic field. Then

$$
n_{+}(s ; p(b) U p(b))=\Phi_{\beta}(s)(1+o(1)), \quad s \downarrow 0,
$$

where

$$
\begin{aligned}
\Phi_{\beta}(s) & =\Phi_{\beta}\left(s ; \eta, b_{0}\right) \\
& := \begin{cases}\frac{b_{0}}{2 \eta^{1 / \beta}}|\ln s|^{1 / \beta} & \text { if } 0<\beta<1, \\
\frac{1}{\ln \left(1+2 \eta / b_{0}\right)}|\ln s| & \text { if } \beta=1, \\
\frac{\beta}{\beta-1}(\ln |\ln s|)^{-1}|\ln s| & \text { if } 1<\beta<\left(0, e^{-1}\right) .\end{cases}
\end{aligned}
$$

Similarly, the combination of $(3.18)$ with [31, Proposition 3.2 with $q=0$ ] and [31, Proposition 3.2] implies

Lemma 3.5. Let $0 \leq U \in L^{\infty}\left(\mathbb{R}^{2}\right)$. Assume that the support of $U$ is compact, and that there exists a constant $C>0$ such that $U \geq C$ on an open nonempty subset of $\mathbb{R}^{2}$. Let $b$ be an admissible magnetic field. Then

$$
n_{+}(s ; p(b) U p(b))=\Phi_{\infty}(s)(1+o(1)), \quad s \downarrow 0,
$$

where

$$
\Phi_{\infty}(s):=(\ln |\ln s|)^{-1}|\ln s|, \quad s \in\left(0, e^{-1}\right) .
$$


Employing now Lemma 3.3 3.4 or 3.5 , we find that 3.7 immediately entails

Corollary 3.6. Let 3.2 with $m>3$ and 3.3 hold true.

(i) Assume that the hypotheses of Lemma 3.3 hold with $U=W$ and $\alpha=m-1$. Then

$$
\begin{aligned}
\xi\left(-E ; H_{0}-V, H_{0}\right) & =-\frac{b_{0}}{2 \pi}\left|\left\{x \in \mathbb{R}^{2} \mid W(x)>2 \sqrt{E}\right\}\right|(1+o(1)) \\
& =-\Psi_{m-1}\left(2 \sqrt{E} ; u_{0}, b_{0}\right)(1+o(1)), \quad E \downarrow 0,
\end{aligned}
$$

the function $\Psi_{\alpha}$ being defined in (3.14).

(ii) Assume that the hypotheses of Lemma 3.4 hold with $U=W$. Then

$$
\xi\left(-E ; H_{0}-V, H_{0}\right)=-\Phi_{\beta}\left(2 \sqrt{E} ; \eta, b_{0}\right)(1+o(1)), \quad E \downarrow 0, \beta \in(0, \infty),
$$

the function $\Phi_{\beta}$ being defined in 3.19 .

(iii) Assume that the hypotheses of Lemma 3.5 hold with $U=W$. Then

$$
\xi\left(-E ; H_{0}-V, H_{0}\right)=-\Phi_{\infty}(2 \sqrt{E})(1+o(1)), \quad E \downarrow 0,
$$

the function $\Phi_{\infty}$ being defined in 3.20 .

Remark. By (1.6), the results of Corollary 3.6, as well those of Theorem 3.1, concern the asymptotic distribution near the origin of the (negative) discrete spectrum of the operator $H_{0}-V$. Results related to Corollary 3.6(i), concerning perturbations $V$ of power-like decay, can be found in 21] where, similarly to the present article, magnetic fields $\mathbf{B}=(0,0, b)$ of constant direction are considered. Moreover, in [21, the perturbation $V$ is not obliged to be asymptotically homogeneous, the decay rate $m$ is allowed to be any positive number, and two distinct types of asymptotic formulae concerning the case $m \in(0,2)$ and $m \in(2, \infty)$ are deduced, the latter being similar to 3.21. On the other hand, in 21] the function $b$ is assumed to be positive, its derivative is supposed to decay at infinity, and the perturbation $V$ is scalar. Results which extend Lemma 3.5, and are related to Corollary 3.6(iii), are contained in [16].

Next, the combination of Theorem 3.2 with Lemmas 3.33 .5 yields

Corollary 3.7. (i) Let 3.2 with $m>3$ and 3.3 hold true. Assume that the hypotheses of Lemma 3.3 are fulfilled for $U=W$ and $\alpha=m-1$. Then

$$
\begin{aligned}
\xi\left(E ; H_{0} \pm V, H_{0}\right) & = \pm \frac{b_{0}}{2 \pi^{2}} \int_{\mathbb{R}^{2}} \arctan \left((2 \sqrt{E})^{-1} W(x)\right) d x(1+o(1)) \\
& = \pm \frac{1}{2 \cos (\pi /(m-1))} \Psi_{m-1}\left(2 \sqrt{E} ; u_{0}, b_{0}\right)(1+o(1)), \quad E \downarrow 0 .
\end{aligned}
$$


(ii) Let (3.2) with $m>3$ and (3.3) hold true. Suppose in addition that $V$ satisfies (3.1) for some $m_{\perp}>2$ and $m_{3}>2$. Finally, assume that the hypotheses of Lemma 3.4 are fulfilled for $U=W$. Then

$$
\xi\left(E ; H_{0} \pm V, H_{0}\right)= \pm \frac{1}{2} \Phi_{\beta}\left(2 \sqrt{E} ; \eta, b_{0}\right)(1+o(1)), \quad E \downarrow 0, \beta \in(0, \infty) .
$$

(iii) Let the assumptions of (ii) be fulfilled, except that the hypotheses of Lemma 3.4 are replaced by those of Lemma 3.5. Then

$$
\xi\left(E ; H_{0} \pm V, H_{0}\right)= \pm \frac{1}{2} \Phi_{\infty}(2 \sqrt{E})(1+o(1)), \quad E \downarrow 0 .
$$

The main ingredient of the proof of Corollary 3.7 is the estimate

$$
\operatorname{Tr} \arctan \left(s^{-1} \Omega(E)\right)=\operatorname{Tr} \arctan \left(s^{-1} \tilde{\Omega}(E)\right)(1+o(1)), \quad E \downarrow 0, s>0,
$$

where

$$
\tilde{\Omega}(E):=\frac{1}{2 \sqrt{E}} p(b)\left(\begin{array}{rr}
W & 0 \\
0 & 0
\end{array}\right) p(b), \quad E>0,
$$

$W$ being defined in (3.4). Estimate 3.22 is obtained by using the Lifshits-Krein trace formula $(1.5)$ with $f(E)=\arctan E, E \in \mathbb{R}$. Since the argument of the proof of Corollary 3.7 is completely analogous to the one of [15. Corollary 3.2], we omit the details.

Remark. By (1.7), Corollary 3.7 as well as Theorem 3.2 concern the low-energy asymptotics of the scattering phase $\arg \operatorname{det} S\left(H_{0} \pm V, H_{0}\right)$.

Putting together the results of Corollaries 3.6 and 3.7 for negative perturbations, we obtain

Corollary 3.8. Under the assumptions of Corollary 3.7 (i) we have

$$
\lim _{E \downarrow 0} \frac{\xi\left(E ; H_{0}-V, H_{0}\right)}{\xi\left(-E ; H_{0}-V, H_{0}\right)}=\frac{1}{2 \cos (\pi /(m-1))}, \quad m>3,
$$

while under the assumptions of Corollary 3.7(ii)-(iii) we have

$$
\lim _{E \downarrow 0} \frac{\xi\left(E ; H_{0}-V, H_{0}\right)}{\xi\left(-E ; H_{0}-V, H_{0}\right)}=\frac{1}{2} .
$$

Remark. Formulae 3.23 -3.24 could be interpreted as generalized Levinson formulae. We recall that the classical Levinson formula relates the (finite) limiting values as $E \uparrow 0$ and $E \downarrow 0$ of the $\operatorname{SSF} \xi(E ;-\Delta+V ;-\Delta)$ where $\Delta$ is the Laplacian in $\mathbb{R}^{d}, d \geq 1$, and $V: \mathbb{R}^{d} \rightarrow \mathbb{R}$ is a scalar potential which decays fast enough at infinity (see the original work [24] or the survey article [32]). 


\section{§4. Auxiliary results}

\section{§4.1. A representation of the SSF}

In this subsection we introduce a suitable representation of the SSF $\xi\left(E ; H_{0} \pm V, H_{0}\right), E \in(-\infty, \mathcal{C}) \backslash\{0\}$, based on a general abstract result of A. Pushnitski [27].

Assume that $V$ satisfies $(3.3)$ and (3.1). Set

$$
L(\mathbf{x})=\left\{\ell_{j k}(\mathbf{x})\right\}_{j, k=1}^{2}:=V(\mathbf{x})^{1 / 2}, \quad \mathbf{x} \in \mathbb{R}^{3} .
$$

Then for $E<0$ we have

$$
\begin{aligned}
L\left(H_{0}-E\right)^{-1 / 2} & \in S_{\infty}\left(L^{2}\left(\mathbb{R}^{2} ; \mathbb{C}^{2}\right)\right), \\
L\left(H_{0}-E\right)^{-1} & \in S_{2}\left(L^{2}\left(\mathbb{R}^{2} ; \mathbb{C}^{2}\right)\right) .
\end{aligned}
$$

For $z \in \mathbb{C}_{+}:=\{\zeta \in \mathbb{C} \mid \operatorname{Im} \zeta>0\}$, set $T(z):=L\left(H_{0}-z\right)^{-1} L$. By [7] (see also [27, Lemma 4.1]), for almost every $E \in \mathbb{R}$ the operator-norm limit

$$
T(E+i 0):=\mathrm{n}-\lim _{\delta \downarrow 0} T(E+i \delta)
$$

exists, and

$$
\operatorname{Im} T(E+i 0) \in S_{1} .
$$

For trivial reasons the limit in (4.4) exists and 4.5) holds for each $E<0=$ $\inf \sigma\left(H_{0}\right)$. In Corollary 4.5 below we show that this is also true for each $E \in(0, \mathcal{C})$. Hence, by [27, Lemma 2.1], the quantity

$$
\begin{aligned}
& \tilde{\xi}\left(E ; H_{0} \pm V, H_{0}\right) \\
= & \pm \int_{\mathbb{R}} n_{\mp}(1 ; \operatorname{Re} T(E+i 0)+t \operatorname{Im} T(E+i 0)) d \mu(t), \quad E \in(-\infty, \mathcal{C}) \backslash\{0\},
\end{aligned}
$$

with

$$
d \mu(t):=\frac{d t}{\pi\left(1+t^{2}\right)},
$$

is well defined. Arguing as in the proof of [12, Proposition 2.5] (see also [11, Proposition 2.1]), and bearing in mind Proposition 4.2, Corollary 4.3, and Proposition 4.4 below, we easily prove the following

Proposition 4.1. Assume that $V$ satisfies (3.1) with $m_{\perp}>2, m_{3}>1$, and (3.3). Suppose that $b$ is an admissible magnetic filed. Then $\tilde{\xi}\left(\cdot ; H_{0} \pm V, H_{0}\right)$ is bounded on every compact subset of $(-\infty, \mathcal{C}) \backslash\{0\}$, and is continuous on $(-\infty, \mathcal{C}) \backslash(\{0\} \cup$ $\left.\sigma_{\mathrm{pp}}(H \pm V)\right)$. 
Since $V$ satisfies (3.1) with $m_{\perp}>2, m_{3}>1$, relation (1.3) holds true and the $\operatorname{SSF} \xi\left(E ; H_{0} \pm V, H_{0}\right)$ is well defined for almost every $E \in \mathbb{R}$. On the other hand, by [27, Theorem 1.2] we have

$$
\xi\left(E ; H_{0} \pm V, H_{0}\right)=\tilde{\xi}\left(E ; H_{0} \pm V, H_{0}\right)
$$

for almost every $E \in \mathbb{R}$. In this article we identify $\xi\left(E ; H_{0} \pm V, H_{0}\right)$ with $\tilde{\xi}(E$; $\left.H_{0} \pm V, H_{0}\right)$ for $E \in(-\infty, \mathcal{C}) \backslash\{0\}$

Remark. The representation of the SSF described above admits a generalization to non-sign-definite perturbations $V$ (see [17, 28]). This generalization is based on the concept of the index of orthogonal projections (see [4]).

We formulate our main results and their corollaries for the case of perturbations of constant sign because certain key auxiliary facts are known to be true only in this case.

\section{§4.2. Estimates of sandwiched resolvents}

For $z \in \mathbb{C}_{+}$define the operator $R(z):=\left(-d^{2} / d x_{3}^{2}-z\right)^{-1}$, bounded in $L^{2}(\mathbb{R})$. The operator $R(z)$ admits the integral kernel $\mathcal{R}_{z}\left(x_{3}-x_{3}^{\prime}\right)$ where $\mathcal{R}_{z}(x)=$ $i e^{i \sqrt{z}|x|} /(2 \sqrt{z}), x \in \mathbb{R}$, and the branch of $\sqrt{z}$ is chosen so that $\operatorname{Im} \sqrt{z}>0$.

For $z \in \mathbb{C}_{+}$introduce the operators

$$
T_{<}(z):=L \mathbf{P}\left(H_{0}-z\right)^{-1} L, \quad T_{>}(z):=L \mathbf{Q}\left(H_{0}-z\right)^{-1} L,
$$

bounded in $L^{2}\left(\mathbb{R}^{3} ; \mathbb{C}^{2}\right)$ (see 2.23 for the definition of the orthogonal projections $\mathbf{P}$ and $\mathbf{Q})$. Then we have $T_{<}(z)=L((p \otimes R(z)) \oplus 0) L$.

For $E \in \mathbb{R}, E \neq 0$, define $R(E)$ to be the operator with the integral kernel $\mathcal{R}_{E}\left(x_{3}-x_{3}^{\prime}\right)$ where

$$
\mathcal{R}_{E}(x):=\lim _{\delta \downarrow 0} \mathcal{R}_{E+i \delta}(x)=\left\{\begin{array}{ll}
\frac{e^{-\sqrt{-E}|x|}}{2 \sqrt{-E}} & \text { if } E<0, \\
\frac{i e^{i \sqrt{E}|x|}}{2 \sqrt{E}} & \text { if } E>0,
\end{array} \quad x \in \mathbb{R} .\right.
$$

For $E \in \mathbb{R}, E \neq 0$, set

$$
T_{<}(E):=L((p \otimes R(E)) \oplus 0) L .
$$

Proposition 4.2. Let (3.1) with $m_{\perp}>2, m_{3}>1$ and $(3.3)$ hold true. Then the operator-valued function $\overline{\overline{\mathbb{C}_{+}}} \backslash\{0\} \ni z \mapsto T_{<}(z) \in S_{1}$ is well defined and continuous. Moreover,

$$
\left\|T_{<}(E)\right\|_{1} \leq C_{1}\left(1+E_{+}^{1 / 4}\right)|E|^{-1 / 2}, \quad E \in \mathbb{R} \backslash\{0\}
$$

with $C_{1}$ independent of $E$. 
Proof. The operator $T_{<}(z)$ admits the representation

$$
T_{<}(z)=M((G \otimes J(z)) \oplus 0) M, \quad z \in \overline{\mathbb{C}_{+}} \backslash\{0\}
$$

where $M: L^{2}\left(\mathbb{R}^{3} ; \mathbb{C}^{2}\right) \rightarrow L^{2}\left(\mathbb{R}^{3} ; \mathbb{C}^{2}\right)$ is multiplication by the matrix-valued function

$$
M\left(x, x_{3}\right):=\langle x\rangle^{m_{\perp} / 2}\left\langle x_{3}\right\rangle^{m_{3} / 2} L\left(x, x_{3}\right), \quad\left(x, x_{3}\right) \in \mathbb{R}^{3}
$$

the operator $G:=\langle x\rangle^{-m_{\perp} / 2} p\langle x\rangle^{-m_{\perp} / 2}$ acts in $L^{2}\left(\mathbb{R}^{2}\right)$, while

$$
J(z):=\left\langle x_{3}\right\rangle^{-m_{3} / 2} R(z)\left\langle x_{3}\right\rangle^{-m_{3} / 2}
$$

acts in $L^{2}(\mathbb{R})$. Evidently,

$$
\left\|T_{<}(z)\right\|_{1} \leq\|M\|^{2}\|G\|_{1}\|J(z)\|_{1}, \quad z \in \overline{\mathbb{C}_{+}} \backslash\{0\} .
$$

By (3.1), the operator $M$ is bounded. Further, $\|G\|_{1}=\|p U p\|_{1}$ with $U(x)=$ $\langle x\rangle^{-m_{\perp}}, x \in \mathbb{R}^{2}$. As $m_{\perp}>2$ we have $U \in L^{1}\left(\mathbb{R}^{2}\right)$, and Lemma 2.4 implies $G \in S_{1}$. Moreover, $M$ and $G$ are independent of $z$. By [12, Subsection 4.1] the operatorvalued function $\overline{\mathbb{C}_{+}} \backslash\{0\} \ni z \mapsto J(z) \in S_{1}$ is well defined and continuous, and admits the estimate

$$
\|J(E)\|_{1} \leq C_{1}^{\prime}\left(1+E_{+}^{1 / 4}\right)|E|^{-1 / 2}, \quad E \in \mathbb{R} \backslash\{0\},
$$

with $C_{1}^{\prime}$ independent of $E$. Now the claim of the lemma follows from 4.104.13 .

For further reference we state the following obvious

Corollary 4.3. Let $V$ satisfy the assumptions of Proposition 4.2 , Let $E \in \mathbb{R}$, $E \neq 0$. Then $\operatorname{Im} T_{<}(E) \geq 0$. Moreover, if $E<0$, then $\operatorname{Im} T_{<}(E)=0$.

Proposition 4.4. Let $V$ satisfy the assumptions of Proposition 4.2. Then the function $\mathbb{C} \backslash[\mathcal{C}, \infty) \ni z \mapsto T_{>}(z) \in S_{2}$ is well defined and analytic. Moreover, for $E \in(-\infty, \mathcal{C})$ we have

$$
T_{>}(E)=T_{>}(E)^{*},
$$

and

$$
\left\|T_{>}(E)\right\|_{2} \leq C_{2}\left(1+\frac{(E+1)_{+}}{\mathcal{C}-E}\right),
$$

with $C_{2}$ independent of $E$. 
Proof. We have

$$
T_{>}(z)=L\left(\left(Q\left(H_{0}^{-}-z\right)^{-1}\right) \oplus\left(H_{0}^{+}-z\right)^{-1}\right) L, \quad z \in \mathbb{C} \backslash[\mathcal{C}, \infty) .
$$

The function $\mathbb{C} \backslash[\mathcal{C}, \infty) \ni z \mapsto T_{>}(z) \in \mathcal{B}$ (the class of bounded linear operators) is well defined and analytic, and (4.14) holds true for $E \in(-\infty, \mathcal{C})$, just because $\mathbb{C} \backslash[\mathcal{C}, \infty)$ is included in the resolvent sets of the operator $H_{0}^{-}$defined on $Q D\left(H_{0}^{-}\right)$, and of the operator $H_{0}^{+}$defined on $D\left(H_{0}^{+}\right)$. Further, set

$$
F\left(x, x_{3}\right)=\langle x\rangle^{-m_{\perp} / 2}\left\langle x_{3}\right\rangle^{-m_{3} / 2}, \quad\left(x, x_{3}\right) \in \mathbb{R}^{3} .
$$

Note that $L=F M$, the matrix $M$ being defined in 4.11). Then we have

$$
\left\|T_{>}(z)\right\|_{2}^{2} \leq\|L\|^{2}\left(\left\|Q\left(H_{0}^{-}-z\right)^{-1} F\right\|_{2}^{2}+\left\|\left(H_{0}^{+}-z\right)^{-1} F\right\|_{2}^{2}\right)\|M\|^{2} .
$$

Applying the spectral theorem for bounded functions of self-adjoint operators, the resolvent identity, and the diamagnetic inequality for Hilbert-Schmidt operators, we get

$$
\begin{aligned}
\left\|Q\left(H_{0}^{-}-z\right)^{-1} F\right\|_{2} & \leq C(z)\left\|\left(H_{0}^{-}+1\right)^{-1} F\right\|_{2} \\
& \leq C(z)\left\|1+\left(H_{0}^{-}+1\right)^{-1} b\right\|\left\|\left((i \nabla+\mathbf{A})^{2}+1\right)^{-1} F\right\|_{2} \\
& \leq C(z)\left\|1+\left(H_{0}^{-}+1\right)^{-1} b\right\|\left\|(-\Delta+1)^{-1} F\right\|_{2}
\end{aligned}
$$

where

Similarly,

$$
C(z):=\sup _{s \in[\mathcal{C}, \infty)}\left|\frac{s+1}{s-z}\right|, \quad z \in \mathbb{C} \backslash[\mathcal{C}, \infty)
$$

$$
\left\|\left(H_{0}^{+}-z\right)^{-1} F\right\|_{2} \leq C(z)\left\|1-\left(H_{0}^{+}+1\right)^{-1} b\right\|\left\|(-\Delta+1)^{-1} F\right\|_{2} .
$$

Since $\left\|(-\Delta+1)^{-1} F\right\|_{2}<\infty$, we find that 4.16-4.18 imply that $T_{>}(z) \in S_{2}$ if $z \in \mathbb{C} \backslash[\mathcal{C}, \infty)$, and that 4.15 holds true.

The analyticity of $T_{>}(z)$ in $S_{2}$ follows from an appropriate estimate of the Hilbert-Schmidt norm of the derivative $d T_{>}(z) / d z$.

Propositions 4.2 and 4.4 immediately entail

Corollary 4.5. Let $V$ satisfy the assumptions of Proposition 4.2 . Then for $E=$ $(-\infty, \mathcal{C}) \backslash\{0\}$ the operator-norm limit 4.4 exists, and

$$
T(E+i 0)=T_{<}(E)+T_{>}(E) .
$$

Moreover,

$$
\begin{aligned}
& \operatorname{Re} T(E+i 0)=\operatorname{Re} T_{<}(E)+T_{>}(E), \\
& \operatorname{Im} T(E+i 0)=\operatorname{Im} T_{<}(E) .
\end{aligned}
$$




\section{$\S 5$. Proofs of the main results}

\section{§5.1. A preliminary estimate}

This subsection contains a preliminary estimate (see $(5.2)$ below) which will be used in the proofs of Theorems 3.13 .2

The following lemma contains a suitable version of the Weyl inequalities for the eigenvalues of compact operators.

Lemma 5.1 ([9, Chapter I, Eq. (1.32)]). Let $T_{j}^{*}, j=1,2$, be compact self-adjoint operators acting in the same Hilbert space. Then

$$
n_{ \pm}\left(s_{1}+s_{2} ; T_{1}+T_{2}\right) \leq n_{ \pm}\left(s_{1} ; T_{1}\right)+n_{ \pm}\left(s_{2} ; T_{2}\right)
$$

for every $s_{1}>0$ and $s_{2}>0$.

Proposition 5.2. Let (3.1) with $m>3$ and (3.3) hold true. Let $E=$ $(-\infty, \mathcal{C}) \backslash\{0\}$. Then the asymptotic estimates

$$
\begin{aligned}
\int_{\mathbb{R}} n_{ \pm}(1+\varepsilon ; \operatorname{Re} & \left.T_{<}(E)+t \operatorname{Im} T_{<}(E)\right) d \mu(t)+O(1) \\
\leq & \int_{\mathbb{R}} n_{ \pm}(1 ; \operatorname{Re} T(E+i 0)+t \operatorname{Im} T(E+i 0)) d \mu(t) \\
\leq & \int_{\mathbb{R}} n_{ \pm}\left(1-\varepsilon ; \operatorname{Re} T_{<}(E)+t \operatorname{Im} T_{<}(E)\right) d \mu(t)+O(1)
\end{aligned}
$$

hold as $E \rightarrow 0$ for each $\varepsilon \in(0,1)$.

Proof. By 4.20 and 4.21, and the Weyl inequalities (5.1), we have

$$
\begin{aligned}
\int_{\mathbb{R}} n_{ \pm} & \left(1+\varepsilon ; \operatorname{Re} T_{<}(E)+t \operatorname{Im} T_{<}(E)\right) d \mu(t)-n_{\mp}\left(\varepsilon ; T_{>}(E)\right) \\
& \leq \int_{\mathbb{R}} n_{ \pm}(1 ; \operatorname{Re} T(E+i 0)+t \operatorname{Im} T(E+i 0)) d \mu(t) \\
& \leq \int_{\mathbb{R}} n_{ \pm}\left(1-\varepsilon ; \operatorname{Re} T_{<}(E)+t \operatorname{Im} T_{<}(E)\right) d \mu(t)+n_{ \pm}\left(\varepsilon ; T_{>}(E)\right) .
\end{aligned}
$$

Evidently, $n_{ \pm}\left(\varepsilon ; T_{>}(E)\right) \leq \varepsilon^{-2}\left\|T_{>}(E)\right\|_{2}^{2}$, which combined with 4.15 yields

$$
n_{ \pm}\left(\varepsilon ; T_{>}(E)\right)=O(1), \quad E \rightarrow 0 .
$$

Now (5.2) follows from $(5.3)$ and $(5.4)$. 


\section{§5.2. Proof of Theorem 3.1}

Throughout the subsection we assume the hypotheses of Theorem 3.1. By Corollary 4.3 we have $\operatorname{Im} T_{<}(-E)=0$, and hence $\operatorname{Re} T_{<}(-E)=T_{<}(-E)$ if $E>0$. Therefore,

$$
\begin{aligned}
\int_{\mathbb{R}} n_{+}\left(s ; \operatorname{Re} T_{<}(-E)+t \operatorname{Im} T_{<}(-E)\right) d \mu(t) & \\
& =n_{+}\left(s ; T_{<}(-E)\right), \quad E>0, s>0 .
\end{aligned}
$$

For $E>0$ define $\mathcal{O}(E): L^{2}\left(\mathbb{R}^{3} ; \mathbb{C}^{2}\right) \rightarrow L^{2}\left(\mathbb{R}^{3} ; \mathbb{C}^{2}\right)$ as the operator with matrixvalued integral kernel

$$
\frac{1}{2 \sqrt{E}} \ell_{j 1}\left(x, x_{3}\right) \mathcal{P}_{b}\left(x, x^{\prime}\right) \ell_{1 k}\left(x^{\prime}, x_{3}^{\prime}\right), \quad j, k=1,2,\left(x, x_{3}\right),\left(x^{\prime}, x_{3}^{\prime}\right) \in \mathbb{R}^{3} .
$$

Proposition 5.3. For each $\varepsilon \in(0,1)$ and $s>0$ we have

$$
\begin{aligned}
n_{+}((1+\varepsilon) s ; \mathcal{O}(E))+O(1) & \leq n_{+}\left(s ; T_{<}(-E)\right) \\
& \leq n_{+}((1-\varepsilon) s ; \mathcal{O}(E))+O(1), \quad E \downarrow 0 .
\end{aligned}
$$

Proof. Fix $s>0$ and $\varepsilon \in(0,1)$. By the Weyl inequalities (5.1),

$$
\begin{aligned}
& n_{+}((1+\varepsilon) s ; \mathcal{O}(E))-n_{-}\left(\varepsilon s ; T_{<}(-E)-\mathcal{O}(E)\right) \\
& \quad \leq n_{+}\left(s ; T_{<}(-E)\right) \leq n_{+}((1-\varepsilon) s ; \mathcal{O}(E))+n_{+}\left(\varepsilon s ; T_{<}(-E)-\mathcal{O}(E)\right) .
\end{aligned}
$$

In order to get (5.6), it suffices to show that there exists a compact operator $\tilde{T}$ such that

$$
\mathrm{n}-\lim _{E \downarrow 0}\left(T_{<}(-E)-\mathcal{O}(E)\right)=\tilde{T}
$$

Pick $m^{\prime} \in(3, m)$ and note that

$$
T_{<}(-E)-\mathcal{O}(E)=\tilde{M}_{m, m^{\prime}}\left(\left(\tilde{G}_{m-m^{\prime}} \otimes \tilde{J}_{m^{\prime}}(E)\right) \oplus 0\right) \tilde{M}_{m, m^{\prime}}
$$

where $\tilde{M}_{m, m^{\prime}}$ is multiplication by the bounded matrix-valued function

$$
\langle x\rangle^{\left(m-m^{\prime}\right) / 2}\left\langle x_{3}\right\rangle^{m^{\prime} / 2} L\left(x, x_{3}\right), \quad\left(x, x_{3}\right) \in \mathbb{R}^{3},
$$

$\tilde{G}_{m-m^{\prime}}: L^{2}\left(\mathbb{R}^{2}\right) \rightarrow L^{2}\left(\mathbb{R}^{2}\right)$ is the operator with integral kernel

$$
\langle x\rangle^{-\left(m-m^{\prime}\right) / 2} \mathcal{P}_{b}\left(x, x^{\prime}\right)\left\langle x^{\prime}\right\rangle^{-\left(m-m^{\prime}\right) / 2}, \quad x, x^{\prime} \in \mathbb{R}^{2},
$$

and $\tilde{J}_{m^{\prime}}(E), E>0$, is the operator with integral kernel

$$
-\frac{1}{2 \sqrt{E}}\left\langle x_{3}\right\rangle^{-m^{\prime} / 2}\left(1-e^{-\sqrt{E}\left|x_{3}-x_{3}^{\prime}\right|}\right)\left\langle x_{3}^{\prime}\right\rangle^{-m^{\prime} / 2}, \quad x_{3}, x_{3}^{\prime} \in \mathbb{R} .
$$


Set

$$
\tilde{T}=\tilde{M}_{m, m^{\prime}}\left(\left(\tilde{G}_{m-m^{\prime}} \otimes \tilde{J}_{m^{\prime}}(0)\right) \oplus 0\right) \tilde{M}_{m, m^{\prime}}
$$

where $\tilde{J}_{m^{\prime}}(0): L^{2}(\mathbb{R}) \rightarrow L^{2}(\mathbb{R})$ is the operator with integral kernel

$$
-\frac{1}{2}\left\langle x_{3}\right\rangle^{-m^{\prime} / 2}\left|x_{3}-x_{3}^{\prime}\right|\left\langle x_{3}^{\prime}\right\rangle^{-m^{\prime} / 2}, \quad x_{3}, x_{3}^{\prime} \in \mathbb{R} .
$$

Note that $\tilde{T}$ admits a matrix-valued integral kernel

$$
-\frac{1}{2} \ell_{j 1}\left(x, x_{3}\right)\left|x_{3}-x_{3}^{\prime}\right| \mathcal{P}_{b}\left(x, x^{\prime}\right) \ell_{1 k}\left(x^{\prime}, x_{3}^{\prime}\right), j, k=1,2,\left(x, x_{3}\right),\left(x^{\prime}, x_{3}^{\prime}\right) \in \mathbb{R}^{3} .
$$

Since $m-m^{\prime}>0$, the operator $\tilde{G}_{m-m^{\prime}}$ is compact by Lemma 2.4 . Since $m^{\prime}>3$ we have $\tilde{J}_{m^{\prime}}(E) \in S_{2}$ for $E \geq 0$. Bearing in mind that $\tilde{M}_{m, m^{\prime}}$ is bounded, we find that the operator $\tilde{T}$ is compact. Finally, $\lim _{E \downarrow 0}\left\|\tilde{J}_{m^{\prime}}(E)-\tilde{J}_{m^{\prime}}(0)\right\|_{2}=0$, which easily implies (5.7).

Proposition 5.4. For each $E>0$ and $s>0$ we have

$$
n_{+}(s ; \mathcal{O}(E))=n_{+}(s ; \omega(E)),
$$

the operator $\omega(E)$ being defined in 3.6.

Proof. Define the operator $K: L^{2}\left(\mathbb{R}^{3} ; \mathbb{C}^{2}\right) \rightarrow L^{2}\left(\mathbb{R}^{2}\right)$ by

$$
(K \mathbf{u})(x):=\sum_{k=1,2} \int_{\mathbb{R}^{2}} \int_{\mathbb{R}} \mathcal{P}_{b}\left(x, x^{\prime}\right) \ell_{1 k}\left(x^{\prime}, x_{3}^{\prime}\right) u_{k}\left(x^{\prime}, x_{3}^{\prime}\right) d x_{3}^{\prime} d x^{\prime}, \quad x \in \mathbb{R}^{2},
$$

where $\mathbf{u}=\left(\begin{array}{l}u_{1} \\ u_{2}\end{array}\right) \in L^{2}\left(\mathbb{R}^{3} ; \mathbb{C}^{2}\right)$. We have

$$
\mathcal{O}(E)=\frac{1}{2 \sqrt{E}} K^{*} K, \quad \omega(E)=\frac{1}{2 \sqrt{E}} K K^{*} .
$$

Since $n_{+}\left(s ; K^{*} K\right)=n_{+}\left(s ; K K^{*}\right)$ for each $s>0$, we get 5.10 .

Putting together (4.6), 5.2), 5.5), 5.6), and 5.10), we get (3.7), which concludes the proof of Theorem 3.1 .

\section{§5.3. Proof of Theorem 3.2}

Throughout this subsection we assume the hypotheses of Theorem 3.2 .

Proposition 5.5. For each $s>0$ we have

$$
n_{ \pm}\left(s ; \operatorname{Re} T_{<}(E)\right)=O(1), \quad E \downarrow 0 .
$$


Proof. The operator $\operatorname{Re} T_{<}(E)$ admits the matrix-valued integral kernel

$$
\begin{aligned}
& -\ell_{j 1}\left(x, x_{3}\right) \frac{\sin \left(\sqrt{E}\left|x_{3}-x_{3}^{\prime}\right|\right)}{2 \sqrt{E}} \mathcal{P}_{b}\left(x, x^{\prime}\right) \ell_{1 k}\left(x^{\prime}, x_{3}^{\prime}\right), \\
& \quad j, k=1,2,\left(x, x_{3}\right),\left(x^{\prime}, x_{3}^{\prime}\right) \in \mathbb{R}^{3} .
\end{aligned}
$$

Arguing as in the proof of Proposition 5.3. we find that n- $\lim _{E \downarrow 0} \operatorname{Re} T_{<}(E)=\tilde{T}$ (see (5.8)-(5.9) ), which implies (5.11).

Making use of Propositions 5.2 and 5.5 and Corollary 4.3 , as well as of the Weyl inequalities (5.1) and the evident identities

$$
\int_{\mathbb{R}} n_{ \pm}(s ; t T) d \mu(t)=\frac{1}{\pi} \operatorname{Tr} \arctan \left(s^{-1} T\right), \quad s>0,
$$

with $T=T^{*} \geq 0, T \in S_{1}$, we obtain the following

Corollary 5.6. For each $\varepsilon \in(0,1)$ and $s>0$ we have

$$
\begin{aligned}
\frac{1}{\pi} \operatorname{Tr} \arctan & \left((s(1+\varepsilon))^{-1} \operatorname{Im} T_{<}(E)\right)+O(1) \\
& \leq \int_{\mathbb{R}} n_{ \pm}\left(s ; \operatorname{Re} T_{<}(E)+t \operatorname{Im} T_{<}(E)\right) d \mu(t) \\
& \leq \frac{1}{\pi} \operatorname{Tr} \arctan \left((s(1-\varepsilon))^{-1} \operatorname{Im} T_{<}(E)\right)+O(1), \quad E \downarrow 0 .
\end{aligned}
$$

Proposition 5.7. For each $E>0$ and $s>0$ we have

$$
n_{+}\left(s ; \operatorname{Im} T_{<}(E)\right)=n_{+}(s ; \Omega(E)),
$$

the operator $\Omega(E)$ being defined in 3.9 . Consequently,

$$
\operatorname{Tr} \arctan \left(s^{-1} \operatorname{Im} T_{<}(E)\right)=\operatorname{Tr} \arctan \left(s^{-1} \Omega(E)\right), \quad E>0, s>0 .
$$

Proof. The operator $\operatorname{Im} T_{<}(E)$ admits the matrix-valued integral kernel

$$
\begin{aligned}
\ell_{j 1}\left(x, x_{3}\right) \frac{\cos \left(\sqrt{E}\left(x_{3}-x_{3}^{\prime}\right)\right)}{2 \sqrt{E}} \mathcal{P}_{b}\left(x, x^{\prime}\right) \ell_{1 k}\left(x^{\prime}, x_{3}^{\prime}\right), & \\
\quad j, k & =1,2,\left(x, x_{3}\right),\left(x^{\prime}, x_{3}^{\prime}\right) \in \mathbb{R}^{3} .
\end{aligned}
$$

Define the operator $\mathcal{K}: L^{2}\left(\mathbb{R}^{3} ; \mathbb{C}^{2}\right) \rightarrow L^{2}\left(\mathbb{R}^{2} ; \mathbb{C}^{2}\right)$ by

$$
\mathcal{K} \mathbf{u}:=\mathbf{v}=\left(\begin{array}{l}
v_{1} \\
v_{2}
\end{array}\right) \in L^{2}\left(\mathbb{R}^{2} ; \mathbb{C}^{2}\right), \quad \mathbf{u}=\left(\begin{array}{l}
u_{1} \\
u_{2}
\end{array}\right) \in L^{2}\left(\mathbb{R}^{3} ; \mathbb{C}^{2}\right),
$$


where

$$
\begin{aligned}
& v_{1}(x):=\sum_{k=1,2} \int_{\mathbb{R}^{2}} \int_{\mathbb{R}} \mathcal{P}_{b}\left(x, x^{\prime}\right) \cos \left(\sqrt{E} x_{3}^{\prime}\right) \ell_{1 k}\left(x^{\prime}, x_{3}^{\prime}\right) u_{k}\left(x^{\prime}, x_{3}^{\prime}\right) d x_{3}^{\prime} d x^{\prime}, \\
& v_{2}(x):=\sum_{k=1,2} \int_{\mathbb{R}^{2}} \int_{\mathbb{R}} \mathcal{P}_{b}\left(x, x^{\prime}\right) \sin \left(\sqrt{E} x_{3}^{\prime}\right) \ell_{1 k}\left(x^{\prime}, x_{3}^{\prime}\right) u_{k}\left(x^{\prime}, x_{3}^{\prime}\right) d x_{3}^{\prime} d x^{\prime}, \quad x \in \mathbb{R}^{2} .
\end{aligned}
$$

We have

$$
\operatorname{Im} T_{<}(E)=\frac{1}{2 \sqrt{E}} \mathcal{K}^{*} \mathcal{K}, \quad \Omega(E)=\frac{1}{2 \sqrt{E}} \mathcal{K} \mathcal{K}^{*} .
$$

Since $n_{+}\left(s ; \mathcal{K}^{*} \mathcal{K}\right)=n_{+}\left(s ; \mathcal{K} \mathcal{K}^{*}\right)$ for each $s>0$, we get 5.13.

Now the combination of (4.6), (5.2), (5.12), and (5.14) yields (3.10).

\section{Acknowledgements}

The author thanks Rafael Tiedra de Aldecoa for an illuminating discussion on the contents of [2].

The partial support of the Chilean Science Foundation Fondecyt under Grant 1090467, and of Núcleo Cientifico ICM P07-027-F "Mathematical Theory of Quantum and Classical Magnetic Systems", is gratefully acknowledged.

\section{References}

[1] S. Alama, P. A. Deift and R. Hempel, Eigenvalue branches of the Schrödinger operator $H-E W$ in a gap of $\sigma(H)$, Comm. Math. Phys. 121 (1989), 291-321. Zbl 0676.47032 MR 0985401

[2] W. O. Amrein, A. M. Boutet de Monvel and V. Georgescu, $C_{0}$-groups, commutator methods and spectral theory of $N$-body Hamiltonians, Progr. Math. 135, Birkhäuser, Basel, 1996. Zbl 0962.47500 MR 1388037

[3] J. Avron, I. Herbst and B. Simon, Schrödinger operators with magnetic fields. I. General interactions, Duke Math. J. 45 (1978), 847-883. Zbl 0399.35029 MR 0518109

[4] J. Avron, R. Seiler and B. Simon, The index of a pair of projections, J. Funct. Anal. 120 (1994), 220-237. Zbl 0822.47033 MR 1262254

[5] J. Bellissard, Gap labelling theorems for Schrödinger operators, in From number theory to physics (Les Houches, 1989), Springer, Berlin, 1992, 538-630. Zbl 0833.47056 MR 1221111

[6] A. Besch, Eigenvalues in spectral gaps of the two-dimensional Pauli operator, J. Math. Phys. 41 (2000), 7918-7931. Zbl 0973.81020 MR 1796817

[7] M. S̆. Birman and S. B. Entina, Stationary approach in abstract scattering theory, Izv. Akad. Nauk SSSR 31 (1967), 401-430 (in Russian); English transl.: Math. USSR-Izv. 1 (1967), 391-420. Zbl 0173.16501 MR 0209895

[8] M. Š. Birman and M. G. Kreĭn, On the theory of wave operators and scattering operators, Dokl. Akad. Nauk SSSR 144 (1962), 475-478 (in Russian); English transl.: Soviet Math. Dokl. 3 (1962), 740-744. Zbl 0196.45004 MR 0139007

[9] M. Š. Birman and M. Z. Solomjak, Quantitative analysis in Sobolev imbedding theorems and applications to spectral theory, Amer. Math. Soc. Transl. (2) 114, Amer. Math. Soc., Providence RI, 1980. Zbl 0426.46020 MR 0562305 
[10] J. F. Bony, V. Bruneau and G. D. Raikov, Resonances and spectral shift function near Landau levels, Ann. Inst. Fourier (Grenoble) 57 (2007), 629-671. Zbl 1129.35053 MR 2310953

[11] Ph. Briet, G. Raikov and E. Soccorsi, Spectral properties of a magnetic quantum Hamiltonian in a strip, Asymptot. Anal. 58 (2008), 127-155. Zbl 1163.35446 MR 2456460

[12] V. Bruneau, A. Pushnitski and G. Raikov, Spectral shift function in strong magnetic fields, Algebra i Analiz 16 (2004), 207-238 (in Russian); English transl.: St. Petersburg Math. J. 16 (2005), 181-209. Zbl 1082.35115 MR 2069004

[13] S. Doi, A. Iwatsuka and T. Mine, The uniqueness of the integrated density of states for the Schrödinger operators with magnetic fields, Math. Z. 237 (2001), 335-371 Zbl 0985.35055 MR 1838315

[14] B. A. Dubrovin and S. P. Novikov, Fundamental states in a periodic field. Magnetic Bloch functions and vector bundles, Dokl. Akad. Nauk SSSR 253 (1980), 1293-1297 (in Russian); English transl.: Soviet Math. Dokl. 22 (1980), 240-244. Zbl 0489.46055 MR 0583789

[15] C. Fernández and G. Raikov, On the singularities of the magnetic spectral shift function at the Landau levels, Ann. Henri Poincaré 5 (2004), 381-403. Zbl 1062.81043 MR 2057679

[16] N. Filonov and A. Pushnitski, Spectral asymptotics of Pauli operators and orthogonal polynomials in complex domains, Comm. Math. Phys. 264 (2006), 759-772. Zbl 1106.81040 MR 2217290

[17] F. Gesztesy and K. Makarov, The $\Xi$ operator and its relation to Krein's spectral shift function, J. Anal. Math. 81 (2000), 139-183. Zbl 0964.47013 MR 1785280

[18] B. C. Hall, Holomorphic methods in analysis and mathematical physics, in First summer school in analysis and mathematical physics (Cuernavaca Morelos, 1998), Contemp. Math. 260, Amer. Math. Soc., Providence, RI, 2000, 1-59. Zbl $0977.46011 \mid$ MR 1770752

[19] R. Hempel and S. Z. Levendorskii, On eigenvalues in gaps for perturbed magnetic Schrödinger operators, J. Math. Phys. 39 (1998), 63-78. Zbl 0916.47054 MR 1489610

[20] T. Hupfer, H. Leschke, P. Müller and S. Warzel, Existence and uniqueness of the integrated density of states for Schrödinger operators with magnetic fields and unbounded random potentials, Rev. Math. Phys. 13 (2001), 1547-1581. [Zbl 1029.81027 MR 1869817

[21] A. Iwatsuka and H. Tamura, Asymptotic distribution of eigenvalues for Pauli operators with nonconstant magnetic fields, Duke Math. J. 93 (1998), 535-574. Zbl 0948.35091 MR 1626727

[22] M. G. KreĬn, On the trace formula in perturbation theory, Mat. Sb. 33 (1953), 597-626 (in Russian). Zbl 0052.12303 MR 0060742

[23] S. Z. Levendorskii, The asymptotics for the number of eigenvalue branches for the magnetic Schrödinger operator $H-\lambda W$ in a gap of $H$, Math. Z. 223 (1996), 609-625. MR 1421959

[24] N. Levinson, On the uniqueness of the potential in a Schrödinger equation for a given asymptotic phase, Danske Vid. Selsk. Mat.-Fys. Medd. 25 (1949), no. 9, 29 pp. Zbl 0032.20702 MR 0030080

[25] I. M. Lifshits, On a problem in perturbation theory connected with quantum statistics, Uspekhi Mat. Nauk 7 (1952), no. 1, 171-180 (in Russian). Zbl 0046.21203 MR 0049490

[26] S. P. Novikov and A. P. Veselov, Exactly solvable two-dimensional Schrödinger operators and Laplace transformations, in Solitons, geometry, and topology: on the crossroad, Amer. Math. Soc. Transl. 179, Amer. Math. Soc., 1997, 109-132. Zbl 0922.35141 MR 1437160

[27] A. Pushnitskiı̌, A representation for the spectral shift function in the case of perturbations of fixed sign, Algebra i Analiz 9 (1997), 197-213 (in Russian); English transl.: St. Petersburg Math. J. 9 (1998), 1181-1194. MR 1610180

[28] _ The spectral shift function and the invariance principle, J. Funct. Anal. 183 (2001), 269-320. Zbl 0998.47003 MR 1844210 
[29] G. D. Raikov, Eigenvalue asymptotics for the Schrödinger operator with homogeneous magnetic potential and decreasing electric potential. I. Behavior near the essential spectrum tips, Comm. Partial Differential Equations 15 (1990), 407-434; Zbl 0739.35055 MR 1243533 Errata, ibid. 18 (1993), 1977-1979. Zbl 0787.35080 MR 1044429

[30] Spectral asymptotics for the perturbed 2D Pauli operator with oscillating magnetic fields. I. Non-zero mean value of the magnetic field, Markov Process. Related Fields 9 (2003), 775-794. Zbl 1064.35124 MR 2072255

[31] G. D. Raikov and S. Warzel, Quasi-classical versus non-classical spectral asymptotics for magnetic Schrödinger operators with decreasing electric potentials, Rev. Math. Phys. 14 (2002), 1051-1072. Zbl 1033.81038 MR 1939760

[32] D. Robert, Semiclassical asymptotics for the spectral shift function, in Differential operators and spectral theory, Amer. Math. Soc. Transl. (2) 189, Amer. Math. Soc., Providence, RI, 1999, 187-203. Zbl 0922.35108 MR 1730513

[33] D. R. Yafaev, Mathematical scattering theory. General theory, Transl. Math. Monogr. 105, Amer. Math. Soc., Providence, RI, 1992. Zbl 0761.47001 MR 1180965 